\title{
Validação de metodologia analítica para determinação de taninos pelo método de difusão radial
}

\author{
SANTOS, L.G. ${ }^{*}$; PEIXOTO SOBRINHO, T.J.S. ${ }^{;}$CABRAL, D.L.V. ${ }^{\text {; }}$ AMORIM, E.L.C.1 \\ 'Laboratório de Produtos Naturais, Departamento de Ciências Farmacêuticas - Centro de Ciências da Saúde \\ Universidade Federal de Pernambuco - Av. Prof Arthur de Sá, s/n, Cidade Universitária 54740-521 - Recife - PE, \\ Brasil, *Corresponding author: lumagomes_68@hotmail.com
}

\begin{abstract}
RESUMO: A metodologia para doseamento de taninos através de difusão radial desenvolvido por Hagerman (1987) vem sendo utilizada em laboratórios de fitoterápicos devido, principalmente, à sua simplicidade de execução, rapidez e baixo custo; contudo, não há relato na literatura sobre a submissão desta metodologia a um estudo de validação. Baseando-se neste fato, o presente estudo visou validar a metodologia de Difusão Radial para o doseamento de taninos. Todos os parâmetros obrigatórios exigidos pela ANVISA foram avaliados. O método foi considerado linear e com alta sensibilidade de quantificação $(27,72 \mu \mathrm{g} /$ poço). Mostrou-se também robusto e com recuperação aceitável (85,96\%). Os resultados obtidos para repetibilidade (intra-corrida) e precisão intermediária (inter-corridas), certificaram a precisão do método, obtendo-se valores entre 1,89 e $7,03 \%$. Para a exatidão, valores entre 100,47 e $105,26 \%$ foram obtidos, os quais se encontram dentro dos limites preconizados pela ANVISA. O método foi considerado preciso, exato e reprodutível, além de ser de fácil execução e de baixo custo.
\end{abstract}

Palavras-chaves: difusão radial, doseamento de taninos, validação de método, quantificação.

\begin{abstract}
Validation of the analytical methodology for the determination of tannins by radial diffusion method. The method to determine tannins by radial diffusion developed by Hagerman (1987) has been explicitly used in herbal laboratories mainly because of its simplicity to implement, speed and low cost; yet, there are no reports in the literature on the submission of this method to a validation study. Based on this fact, this study sought to validate the method of Radial Diffusion in the tannin determination. All mandatory parameters required by ANVISA were evaluated. The method was considered linear and with high sensitivity quantitation (27.72 $\mu \mathrm{g} / \mathrm{well})$. It also showed robust and acceptable recovery (85.96\%). The results obtained for repeatability (within-run) and intermediate precision (inter-run) certified the accuracy of the method, obtaining values between 1.89 and $7.03 \%$. For accuracy, values between 100.47 and $105.26 \%$ were obtained, which is within the limits recommended by ANVISA. Thus, the method was considered as precise, accurate and reproducible, and is easy to perform and inexpensive.
\end{abstract}

Keywords: radial diffusion, tannin content, method validation, quantification.

\section{INTRODUÇÃO}

Muito se tem falado sobre validação, certificação e qualificação de instalações, equipamentos, processos, e metodologia analítica; porém, a falta de padronização de linguagem do setor cria uma barreira para o completo entendimento dos termos (Athaide, 2000). Milhões de medições analíticas são efetuadas a cada dia em laboratórios ao redor do mundo e verifica-se a necessidade progressiva de dados analíticos comparáveis e consistentes para a eliminação de barreiras técnicas entre os vários países. Para atingir este processo de reconhecimento mútuo a nível internacional, em que uma vez efetuada a medição, esta é aceita em qualquer país, requisitos legais, de certificação e de credenciamento devem ser observados. As normas internacionais, nacionais e sistemas da qualidade destacam a importância da validação de métodos analíticos e a documentação do trabalho de

Recebido para publicação em 26/04/2012

Aceito para publicação em 20/05/2014

10.1590/1983-084X/12_023

Rev. Bras. Pl. Med., Campinas, v.16, n.4, p.881-885, 2014. 
validação, para a obtenção de resultados confiáveis e adequados ao uso pretendido (Barros, 2002).

De acordo com a United States Pharmacopeia XXIV (2000), a validação de métodos analíticos "é o processo pelo qual é estabelecido, por estudos de laboratório, que as características executadas do método satisfazem os requisitos para as aplicações analíticas praticadas", ou seja, é a confirmação por exame e fornecimento de evidência objetiva de que os requisitos específicos para um determinado uso pretendido são atendidos (NBR ISO/IEC 17025, 2001). As características analíticas típicas usadas na validação de métodos são: exatidão, precisão, especificidade, limite de detecção, limite de quantificação, linearidade, intervalo de aceitação, robustez ou resistência e conformidade do sistema (Brasil, 2003; Espires, 1998; Pecora, 2000; Pinto et al., 2003). Portanto, a validação de metodologias analíticas necessita ser especifica, robusta, sensível, precisa e exata, constituindo fundamental importância para o controle de qualidade dos produtos e sendo parte das normas de Boas Práticas de Fabricação e Controle (Barros Neto et al., 2002).

A realização da validação deve ser executada: i) sempre que de forma direta ou indireta quando o processo de fabricação tenha sido alterado; ii) quando a qualidade final do produto for duvidosa; iii) em equipamentos novos e iv) em caso de implantação de um processo ou método analítico novo (Emanuelli, 2000; Pasteelnick, 1993).

O método de difusão radial desenvolvido por Hagerman (1987) para o doseamento de taninos se fundamenta na propriedade que estes metabólitos secundários possuem de se complexar com as proteínas. No ensaio, o tanino difunde por um gel contendo proteína e o precipitado desenvolve uma forma de disco visível à medida que o tanino interage com a proteína. O método é simples, sensível e específico, e deve ser especialmente aplicado para estudos nos quais um grande número de amostras será analisado. Apesar de já bastante utilizado e difundido na comunidade acadêmica, não há relatos de que a metodologia tenha passado por um processo de validação, estudo este de extrema importância para se garantir a confiabilidade do método. Neste sentido, o objetivo deste trabalho foi realizar a validação da metodologia analítica de quantificação de taninos por difusão radial.

\section{MATERIAL E MÉTODO}

\section{Reagentes e equipamentos}

O solvente usado foi o metanol (Vetec Química Fina, 99,8\%). Os reagentes foram ácido acético glacial (Merck, 99,8\%), hidróxido de sódio (Vetec Química Fina, 99\%), ácido ascórbico 99,5\% (Vetec Química Fina), agarose tipo I (Sigma-Aldrich) e albumina sérica bovina fração $V$ livre de ácidos graxos (Sigma-Aldrich). Como padrão para taninos foi utilizado ácido tânico 99,5\% (Vetec Química Fina).

As pesagens foram realizadas em balança analítica Shimadzu AX200 e as diluições com micropipetas monocanal de volume variável HTL Lab Solutions. A bancada de vidro foi nivelada com inclinômetro Insize $\mathrm{C} 7$. As análises de $\mathrm{pH}$ foram realizadas em pHmetro de bancada Tecnopon mPA210.

\section{Preparação do gel}

Foi preparada uma solução $50 \mathrm{mM}$ de ácido acético e $60 \mu \mathrm{M}$ de ácido ascórbico, sendo o pH ajustado para 5,0 pela adição de hidróxido de sódio em pérolas pois este é o $\mathrm{pH}$ ideal para a interação tanino-proteína (López et al., 2004). O gel foi preparado utilizando agarose $(1 \%, p / v)$ na solução descrita previamente. A mistura foi homogeneizada sob aquecimento e, após o resfriamento a $45^{\circ} \mathrm{C}$, foi adicionada albumina sérica bovina $(0,1 \%, \mathrm{p} / \mathrm{v})$. Alíquotas de $10 \mathrm{~mL}$ do gel foram distribuídas em placas de Petri (90 mm x $15 \mathrm{~mm}$ ) nas quais foram feitos poços com o auxílio de um perfurador de 5 $\mathrm{mm}$ de diâmetro. As amostras foram aplicadas nos poços com micropipetas.

\section{Procedimentos experimentais}

Para avaliar a robustez e a recuperação do método, $100 \mathrm{mg}$ da amostra seca e pulverizada de cedro (Cedrela odorata L.), foram pesados e transferidos para tubos de penicilina, sendo adicionado $1 \mathrm{~mL}$ de acetona $50 \%$ para extração durante 60 minutos. Os extratos foram filtrados por aspirados. Deste filtrado, $24 \mu \mathrm{L}$ foram aplicados nos poços.

As placas contendo os halos com amostras vegetais e padrão ácido tânico foram escaneadas e foi utilizado o programa Corel Draw@ X3 Versão 13 para mensuração dos diâmetros dos halos.

\section{Parâmetros da validação}

Para a linearidade foi usada a média de três intervalos com repetições autênticas, que contemplavam seis concentrações da solução de ácido tânico 25 mg/mL (100 - $600 \mu \mathrm{g} /$ poço). Após a relação linear visual, os resultados foram analisados estatisticamente para definir o coeficiente de determinação, a equação de regressão, o ajuste linear e o desvio padrão relativo (Barros Neto et al., 2002; Brasil, 2003). Os limites de detecção e quantificação foram estimados (em $\mu \mathrm{g} /$ poço) considerando o desvio padrão em razão 
ao coeficiente angular (inclinação da reta) obtidos pela linearidade, nos quais foram utilizadas as equações 1 e 2 para determinar o limite de detecção e quantificação, respectivamente (Brasil, 2003).

$$
\begin{aligned}
& \mathrm{LD}=\mathrm{DPa} \times 3 / \mathrm{IC}(1) \\
& \mathrm{LQ}=\mathrm{DPa} \times 10 / \mathrm{IC}(2)
\end{aligned}
$$

Onde o LD é o limite de detecção; LQ é o limite de quantificação; DPa é o desvio-padrão relativo; IC é a inclinação de curva de calibração.

O parâmetro recuperação foi realizado com três amostras em triplicata e o resultado foi obtido através da equação 3. Os fatores a serem considerados para analisar a robustez foram: tempo de extração (60 e 120 minutos) e estabilidade de leitura (3 e 4 dias), o que está de acordo com o preconizado pela ANVISA (Brasil, 2003). Os resultados foram avaliados mediante comparação através da análise de variância.

$$
\mathrm{R}(\%)=\text { CTE/CTNE } \times 100(3)
$$

Onde CTE compreende a concentração total de taninos extraídos (simulação do processo extrativo) e CTNE é a concentração de taninos não extraídos. R (\%) é a recuperação obtida (Brasil, 2003).

Os ensaios de repetibilidade e precisão intermediária foram determinados por seis amostras de mesma concentração, executados no mesmo dia (intra-corrida), e em dois dias consecutivos por analistas diferentes (inter-corrida) (Brasil, 2003). Os resultados foram expressos como desvio padrão relativo (Equação 4). A exatidão foi avaliada por três controles (em sextuplicata) de concentração baixa, média e alta. A exatidão (Equação 5) foi calculada, individualmente para cada controle.

$$
\begin{aligned}
& \mathrm{DPR}(\%)=\mathrm{DP} / \mathrm{CMD} \times 100(4) \\
& \mathrm{E}(\%)=\mathrm{CME} / \mathrm{CT} \times 100(5)
\end{aligned}
$$

Onde o DPR (\%) é a precisão; DP é o desviopadrão; CMD é a concentração média determinada; $E(\%)$ é a exatidão; CME é a concentração média experimental; e CT é a concentração teórica.

\section{RESULTADOS E DISCUSSÃO}

O método validado apresentou linearidade para as concentrações estudadas (100 a 600 g/ poço). A equação da regressão linear média obtida a partir de três curvas de calibração foi $y=0,646 x$ $+36,664$, onde y é o diâmetro quadrado $\left(\mathrm{mm}^{2}\right)$ e $\mathrm{x}$ a concentração ( $\mu \mathrm{g} / \mathrm{poço})$ em equivalentes de ácido tânico. Como mostrado na Figura 1, o coeficiente de determinação obtido foi $\mathrm{R}^{2}=0,9965$, comprovando a adequação do método ao intervalo avaliado (Brasil, 2003). Os dados de precisão (DPR) e exatidão (E) do intervalo do método são apresentados na Tabela 1.

Os valores dos limites de detecção (LD) e quantificação (LQ) estimados pelas equações 1 e 2 , foram 8,32 e 27,72 $\mu \mathrm{g} /$ poço, respectivamente. Com esses resultados, verificamos que o método possui alta sensibilidade para detectar e quantificar

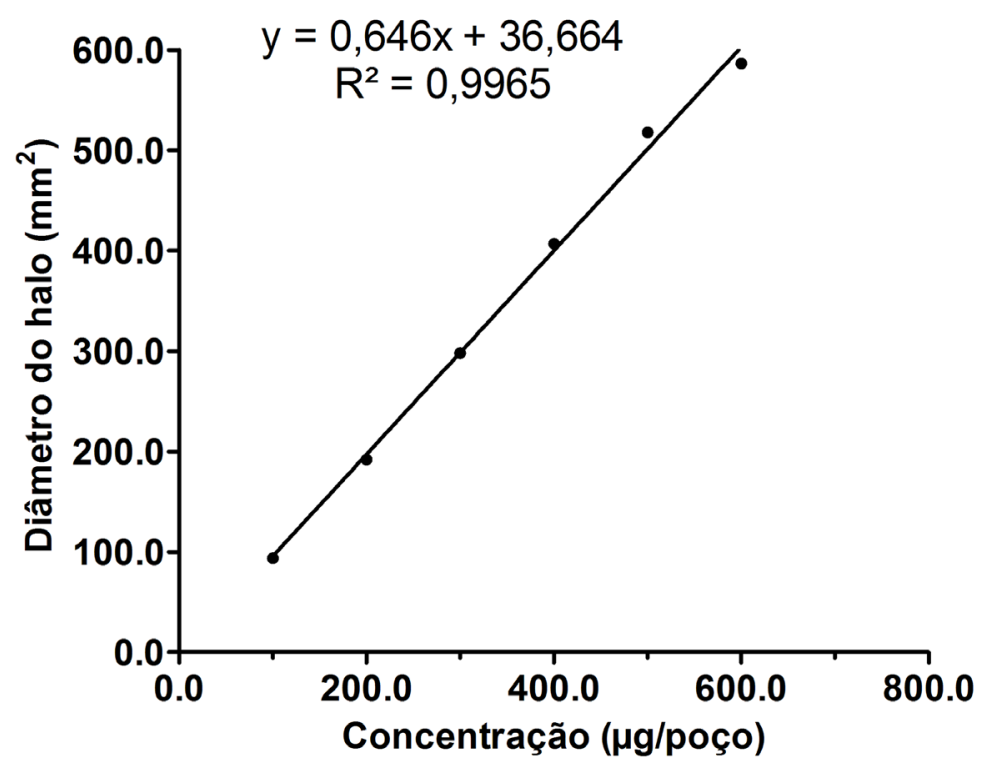

FIGURA 1. Curva de calibração construída com padrão ácido tânico (100 a $600 \mu \mathrm{g} /$ poço) onde a equação linear média obtida foi $y=0,646 x+36,664$ e $R^{2}=0,9965$. 
TABELA 1. Resultados do teste de linearidade por difusão radial para taninos, utilizando-se ácido tânico como padrão.

\begin{tabular}{ccccc}
\hline Concentração teórica $(\mu \mathrm{g} / \mathrm{mL})$ & $C(n=3)$ & $D P$ & $D P R(\%)$ & $E(\%)$ \\
\hline 100 & 93,95 & 2,81 & $2,99 \%$ & $93,95 \%$ \\
200 & 191,92 & 6,29 & $3,28 \%$ & $95,96 \%$ \\
300 & 298,22 & 10,25 & $3,44 \%$ & $99,41 \%$ \\
400 & 406,94 & 7,14 & $1,76 \%$ & $101,74 \%$ \\
500 & 517,95 & 23,58 & $4,55 \%$ & $103,59 \%$ \\
600 & 586,56 & 39,93 & $6,81 \%$ & $97,76 \%$ \\
\hline
\end{tabular}

C = Concentração média ( $\mu \mathrm{g} /$ poço) de três determinações; DP = Desvio-padrão; DPR (\%) = Desvio-Padrão Relativo (Precisão); E (\%) = Exatidão.

o padrão, sem sofrer alteração de fatores intrínsecos do método.

A análise de variância ANOVA confirmou que o método proposto foi considerado linear e indica que não há falta de ajuste $(\mathrm{p}<0,05)$ (Pimentel, Barros Neto, 2002), como pode ser visto na Tabela 2.

A recuperação que mede a eficiência do procedimento de extração do método proposto foi $85,96 \%$ (Brasil, 2003). A análise de variância ANOVA confirmou que o método proposto foi considerado robusto ( $p=0,095)$ (Pimentel, Barros Neto, 2002).

Os dados da repetibilidade, precisão intermediária (intradia e interdia) e exatidão do método encontram-se na Tabela 3.

Os ensaios de repetibilidade (intradia) e precisão intermediária (interdia) estão dentro dos parâmetros exigidos, com valores compreendidos entre 1,89 e $7,03 \%$. Para o ensaio de exatidão foram encontrados resultados entre 100,47 e $105,26 \%$. A ANVISA regulamenta que os resultados da exatidão não devem ser inferiores a 95\% (Brasil, 2003). Estes dados confirmam que o método proposto encontra-se em conformidade com a legislação vigente e apresenta confiabilidade dos resultados. O teste $t$ de Student apontou que não há diferença estatística entre as concentrações determinadas e

TABELA 2. Resultados da Análise de Variância (ANOVA) e teste de ajuste linear $(p<0,05)$ do parâmetro linearidade da validação.

\begin{tabular}{cccccc}
\hline \multicolumn{7}{c}{ ANOVA } \\
\hline Fonte & $\mathrm{SQ}$ & $\mathrm{gL}$ & $\mathrm{MQ}$ & $\mathrm{F}$ & $\mathrm{F}_{\text {Critico }}$ \\
\hline Modelo & 540053,86 & 1 & 540053,86 & 1317,33 & 4,49 \\
\hline Residual & 6559,38 & 16 & 409,96 & \multicolumn{2}{c}{ Curva Linear } \\
\hline Falta de ajuste & 1851,31 & 4 & 462,83 & 1,18 & 3,26 \\
\hline Erro puro & 4708,07 & 12 & 392,34 & Não há falta de ajuste \\
\hline Total & 546613,24 & 17 & 32153,72 & & \\
\hline
\end{tabular}

TABELA 3. Resultados da repetibilidade, precisão intermediária e exatidão do método de quantificação de taninos por difusão radial.

\begin{tabular}{|c|c|c|c|c|c|c|}
\hline Ensaio & \multicolumn{2}{|c|}{$\begin{array}{l}\text { Concentração } \\
\text { teórica ( } \mu \mathrm{g} / \text { poço) }\end{array}$} & C & DP & DPR (\%) & $E(\%)$ \\
\hline Repetibilidade & \multicolumn{2}{|l|}{200,0} & 203,37 & 8,41 & 4,14 & 101,68 \\
\hline \multirow{4}{*}{ Precisão intermediária } & \multirow{2}{*}{ Analista 1} & 200,0 & 203,39 & 8,39 & 4,13 & 101,70 \\
\hline & & 200,0 & 200,94 & 14,12 & 7,03 & 100,47 \\
\hline & \multirow{2}{*}{ Analista 2} & 200,0 & 203,33 & 13,89 & 6,83 & 101,67 \\
\hline & & 200,0 & 210,53 & 6,79 & 3,23 & 105,26 \\
\hline \multirow{3}{*}{ Exatidão } & \multicolumn{2}{|l|}{100,0} & 103,56 & 2,37 & 2,28 & 103,56 \\
\hline & \multicolumn{2}{|l|}{200,0} & 206,24 & 3,90 & 1,89 & 103,12 \\
\hline & \multicolumn{2}{|l|}{300,0} & 309,85 & 9,80 & 3,16 & 103,28 \\
\hline
\end{tabular}

Rev. Bras. PI. Med., Campinas, v.16, n.4, p.881-885, 2014. 
as concentrações teóricas ( $\mu \mathrm{g} /$ poço).

Esta metodologia vem sendo amplamente utilizada em laboratórios de estudos de fitoterápicos, pois representa uma maneira simples e rápida de determinação do teor de taninos de uma determinada espécie ou gênero, em comparação com outras metodologias já existentes, que geralmente são laboriosas, demoradas, empregam produtos químicos que exigem condições especiais de manipulação e, sobretudo, são inespecíficas (Melo et al., 2010).

A maior aplicabilidade do método de difusão radial encontra-se quando da necessidade de análise de um grande número de amostras, requisitada, principalmente, em estudos de triagem para seleção de plantas com potenciais atividades terapêuticas, e os que visam comparação de determinadas condições a que a espécie vegetal está submetida e como estas condições são refletidas no metabolismo secundário da planta (Cabral et al., 2010; Siqueira et al., 2011).

\section{CONCLUSÃO}

O método proposto apresenta alta linearidade e confirma possuir alta sensibilidade de quantificação. A recuperação mostra que aproximadamente $85 \%$ dos taninos presentes na amostra podem ser quantificados por este método. Os resultados de precisão e exatidão obtidos atestam confiabilidade necessária para o uso desta metodologia em laboratórios de controle de qualidade. O método de quantificação por difusão radial mostrou-se preciso, exato e reprodutível, aliado a acessibilidade e facilidade de execução.

\section{AGRADECIMENTOS}

Os autores agradecem a Fundação de Amparo a Ciência e Tecnologia do Estado de Pernambuco (FACEPE) pela bolsa de Doutorado concedida a D.L.V. Cabral e a Coordenação de Aperfeiçoamento de Pessoal do Ensino Superior (CAPES) pela bolsa de Pós-Doutorado concedida a T.J.S. Peixoto Sobrinho e de Mestrado concedida a L. G. Santos, ao Prof. Dr Pedro José Rolim Neto e sua equipe do Laboratório de Tecnologia dos Medicamentos (LTM-UFPE) pelo apoio dado durante todo o estudo.

\section{REFERÊNCIA}

ATHAIDE A. Validação comprova e documenta qualidade dos produtos e equipamentos. Controle de
Contaminação. 2000; maio/jun.:16-22.

BARROS NETO, B.; PIMENTEL, M. F.; ARAÚJO, M. C. U. Recomendações para calibração em química analítica- Parte I. Fundamentos e calibração com um componente (calibração univariada). Quim. Nova, v.25, n.5, p.856- 865, 2002.

BARROS C. B. Validação de métodos analíticos. Biológico, São Paulo, v.64, n.2, p.175-177, jul./dez., 2002.

BRASIL, Agência Nacional de Vigilância Sanitária (ANVISA). R. E, nº 899 de 29 de maio de 2003 - Guia para validação de métodos qualitativos e bioanalíticos.

CABRAL, D.L.V.; PEIXOTO SOBRINHO, T.J.S.; AMORIM, E.L.C.; ALBUQUERQUE, U.P. Relationship of biometric parameters on the concentration of tannins in two medicinal plants - a case study. Boletín Latinoamericano y del Caribe de Plantas Medicinales y Aromáticas, v.9, n.5, p.368-376, 2010.

EMANUELLI T, SCANDIUZZI M. Validação de processos na indústria farmacêutica. In: Congresso de Produtos Farmacêuticos e Cosméticos. 2000; Rio Grande do Sul: Universidade do Rio Grande do Sul. Anais. 2000. p.57.

ESPIRES RC. Aspectos da metodologia analítica do metronidazol. São Paulo: USP, 1998. 136 p. [Dissertação - Mestrado em Ciências Farmacêuticas]. Universidade de São Paulo; 1998.

HAGERMAN, A.E. Radial difusion method for determining tannin in plant extrats. Journal of Chemical Ecology, 13(3): 437-448, 1987.

LÓPEZ, J.; TEJADA, I.; VÁSQUEZ, C.; DE DIOS GARZA, J.; SHIMADA, A. Condensed tannins in tropical fodder crops and their in vitro biological activity: Part 2. Journal of the Science and Food Agriculture, 84: 295-29, 2004.

MELO, J.G.;ARAÚJO, T.A.S.;ALMEIDAE CASTRO, V.T.N.; CABRAL, D.L.V.; RODRIGUES, M.D.; NASCIMENTO, S.C.; AMORIM, E.L.C.; ALBUQUERQUE, U.P. Antiproliferative Activity, Antioxidant Capacity and Tannin Content in Plants of Semi-Arid Northeastern Brazil. Molecules, v.15, p.8534-8542, 2010.

NBR ISO/IEC 17025. Requisitos Gerais para a Competência de Laboratórios de Calibração e de Ensaios. 2001 ABNT. RJ. Brasil.

PASTEELNICK LA. Analytical methods validation. In: Berry IR, Nash RA. Pharmaceutical process validation. New York: Marcel Dekker; 1993. p.411-28.

PECORAM. Validação de métodos analíticos. São Paulo: UNIFAR, 2000. 45p. Apostila (Curso de Validação) União Farmacêutica de São Paulo; 2000.

PINTO TJA, FERRARINI M, GATTI RM. Proposta de roteiro prático para a validação de métodos analíticos. Farmácia \& Química. 2003;36:26-36.

SIQUEIRA, C.F.Q.; CABRAL, D.L.V.; PEIXOTO SOBRINHO, T.J.S.; AMORIM, E.L.C.; MELO, J.G.; ARAÚJO, T.A.S.; ALBUQUERQUE, U.P. Level of Tannins and Flavonoids in Medicinal Plants: Evaluating Bioprospecting Strategies. Evidence-Based Complementary and Alternative Medicine, v.2012, n.2012, 2011.

UNITED STATES PHARMACOPEIA. 24.ed. Rockville U.S.:Pharmacopeial Convention; 2000. p.2149-63. 